\title{
Evaluation of oxacillin and cefoxitin disks for detection of resistance in coagulase negative staphylococci
}

\author{
Ana Lúcia Souza Antunes*/**/+, Carina Secchi, Keli Cristine Reiter, \\ Leandro Reus Rodrigues Perez, Ana Lúcia Peixoto de Freitas, Pedro Alves d'Azevedo
}

Fundação Faculdade Federal de Ciências Médicas de Porto Alegre, Porto Alegre, RS *Faculdade de Farmácia, Universidade Federal do Rio Grande do Sul, 90610-000 Porto Alegre, RS, Brasil **Universidade Católica de Pelotas, Pelotas, RS, Brasil

Coagulase-negative Staphylococcus spp. was considered nonpathogenic until the emergence of multiresistance and the demonstration of their participation as infectious agents. In Brazil, oxacillin resistance may be present in over $80 \%$ of isolates, and the Clinical and Laboratory Standards Institute standardized a diskdiffusion method to predict this resistance in Staphylococcus. The aim of this study was to evaluate the variability among commercial disks of oxacillin $(1 \mu \mathrm{g})$ and cefoxitin $(30 \mu \mathrm{g})$ widely used in clinical laboratories of microbiology, compared with mecA gene and minimum inhibitory concentration (MIC) of oxacillin. The use of oxacillin and cefoxitin disks simultaneously allowed the detection of important differences, particularly, in less frequent species such as S. cohnii, S. haemolyticus, S. saprophyticus, and S. sciuri. Disks of cefoxitin of the brand 2 displayed good correlation with the mecA gene (98.7\%) and oxacillin MIC (97.8\%), while major discrepancies were observed using disks of brand 1. One of the critical points in the diffusion disk test is the quality of the disks: the use of better quality disks associated with molecular methods lead to better results to define the best antibiotic therapy.

Key words: coagulase negative staphylococci - mecA gene - methicillin resistance - cefoxitin - susceptibility tests

Coagulase-negative Staphylococcus spp. (CoNS) were considered nonpathogenic until their implication as nosocomial agents and the emergence of multiresistance (Beekmann et al. 2003). Healthcare-associated infections caused by these microorganisms are responsible for high mortality rates worldwide. The emergence of CoNS populations with heterogeneous resistance to oxacillin causes a great difficulty to detect them in clinical routine laboratories (Cauwelier et al. 2004). In Brazil, oxacillin resistance may be present in over $80 \%$ of isolates in some health institutions (Sader et al. 2001). On this account, vancomycin has been widely used and it is a major cause for the emergence of glycopeptide-resistant isolates (Oliveira et al. 2001, Nunes et al. 2006).

Recently, Clinical and Laboratory Standards Institute (CLSI) standardized a disk-diffusion method to predict resistance mediated by the mecA gene in Staphylococcus spp., using a cefoxitin disk ( $30 \mu \mathrm{g})$. In fact, studies indicate that this is the best phenotypic test to predict resistance to beta-lactam agents among CoNS (Felten et al. 2002, Pottumarthy et al. 2005). Regardless of the CLSI guidelines, the detection of this resistance through phenotypic methods remains a challenge for clinical laboratories of microbiology (Sejas et al. 2003). Several dis-

Financial support: Capes, CNPq, UFRGS, UCPel, FFCMPA

+Corresponding author: analucia2112@gmail.com

Received 26 February 2007

Accepted 2 July 2007 crepancies were observed among the laboratories that participated in the Antimicrobial Surveillance Program (SENTRY), suggesting non-observance of the interpretation criteria currently recommended (Mendes et al. 2003). The quality of the antimicrobial disks may affect the results, with failure to detect hetero-resistance (Sejas et al. 2003). This is especially important in the case of oxacillin and cefoxitin, which predict susceptibility to a large group of antimicrobial agents.

The aim of this study was to evaluate two different brands of oxacillin $(1 \mu \mathrm{g})$ and cefoxitin $(30 \mu \mathrm{g})$ disks that are commonly used in clinical laboratories of microbiology.

\section{MATERIALS AND METHODS}

Isolates - CoNS isolated from blood cultures maintained in "skim-milk" (Difco, Detrot) at $-20^{\circ} \mathrm{C}$ were studied. For the tests, the isolates were cultivated onto agar plates supplemented with sheep blood at 5\% (Trypticasein agar - Oxoid, UK), incubated for $24 \mathrm{~h}$ at $35^{\circ} \mathrm{C}$.

Identification of CoNS - Characterization was made by a set of phenotypic tests (Bannerman 2003): coagulase, catalase, alkaline phosphatase, ornithine decarboxylase, urease, PYR, and utilization of carbohydrates (trehalose, mannitol, mannose, sucrose, maltose, lactose, cellobiose). Anaerobic growth in thioglicolate and susceptibility to novobiocin (>16 mm), to polymyxin B $(\geq 10 \mathrm{~mm})$, and to bacitracin $(>9 \mathrm{~mm})$ by disk diffusion tests were also evaluated. Other tests useful to discriminate between S. epidermidis and S. hominis were susceptibility to desferrioxamine $(\geq 20 \mathrm{~mm})$ and fosfomycin (> $30 \mathrm{~mm}$ ) (Lindsay et al. 1993, Rosco 2000).

Susceptibility tests - Oxacillin (1 $\mu \mathrm{g})$ and cefoxitin $(30 \mu \mathrm{g})$ disks from two different brands, widely used in clinical microbiology laboratories were tested. A 0.5 
Mcfarland standard suspension of each isolate was inoculated onto a Mueller Hinton agar plate (Oxoid), and the disks were distributed maintaining a distance of 30 $\mathrm{mm}$ edge to edge. The diameters of the inhibition zones were interpreted according to the criteria recommended by the CLSI (2006), considering a breakpoint diameter for susceptibility inhibition of $\geq 18 \mathrm{~mm}$ to oxacillin and $\geq 25 \mathrm{~mm}$ to cefoxitin. Using a Steer's replicator apparatus (Craft Machine, Chester, PA, US), a suspension of each isolate was inoculated onto Muller-Hinton agar plates supplemented with $2 \% \mathrm{NaCl}$ in the following conditions: 0.125 to $4 \mu \mathrm{g}$ of oxacillin (Sigma Chemical Co., St. Louis, MO). The tests were interpreted after $24 \mathrm{~h}$ of incubation at $35^{\circ} \mathrm{C}$, and growth of more than one colony was considered a positive result. Both strains $S$. aureus ATCC 29213 (oxacillin susceptible) and S. aureus ATCC 33591 (oxacillin resistant) were included as controls.

Detection of the mecA gene - A polymerase chain reaction (PCR) based on the methodology previously described (Vannuffel et al. 1998) was used to determine the presence of the mecA gene. Briefly, staphylococcal DNA was extracted by boiling (Nunes et al. 1999) followed by PCR using with specific primers: $\operatorname{mec}^{1}: 5$ TGG CTA TCG TGT CAC AAT CG, mecA 2 : 5 CTG GAA CTT GAG CAG AG. A positive result was indicated by observation of the presence of a 310-bp DNA fragment after gel electrophoresis (Vannuffel et al. 1998). $S$. aureus ATCC 25923 (mecA negative) and S. aureus ATCC 33591 (mecA positive) were the controls used in all sets.

\section{RESULTS}

A total of 302 CoNS isolates were identified (Table I). The most frequent CoNS species found was S. epidermidis, with 157 isolates, followed by S. hominis (56 isolates), S. haemolyticus (52 isolates), S. sciuri (10 isolates), and $S$. warneri (9 isolates); other species were found at a lower percentage. The percentage of resistance accordingly to oxacillin minimum inhibitory concentration (MIC) was greater than $90 \%$ in the three prevalent species.

Resistance according to PCR determination of mecA gene was detected in $88.1 \%$ of the cases, comparing to $90.4 \%$ detected by MIC. The distribution of resistance observed with oxacillin and cefoxitin disks of both brands, as well as MIC and mecA results are shown in Table II. Oxacillin MIC and $m e c A$ gene displayed concordant results in resistant isolates. Resistance to oxacillin was detected in 248 cases $(82.1 \%$ ) by brand 1 and in 271 cases $(89.7 \%$ ) by brand 2 disks. Cefoxitin disks of brand 1 showed concordant results in $154(50.9 \%)$ of the resistant isolates while brand 2 detected $264(87.4 \%)$ of them.

Considering only the results obtained with brand 2 , discrepancy between phenotypic and genotypic methods was observed in 14 cases (Table III). The mecA gene was observed in only four isolates, all with MIC compatible with resistance, although all of them displayed disagreement with disks (cefoxitin or oxacillin disk).

Among isolates with negative PCR to mecA gene, two cases confirmed susceptibility through MIC and cefoxitin disk. The other eight isolates without mecA gene are resistant accordingly to MIC. Of them, the one identified as $S$. epidermidis displayed a considerable high level of resistance (MIC > $4 \mu \mathrm{g} / \mathrm{ml}$ ), although considered susceptible to both disks. Susceptibility test with cefoxitin disk agreed in all but one case with negative mecA gene. Disagreement with oxacillin disks were observed in this and in other seven cases.

TABLE I

Prevalence and resistance of Staphylococcus species

\begin{tabular}{lrcc}
\hline Species & $\mathrm{n}$ & Total & $\begin{array}{c}\text { \% resistance } \\
(\text { mecA) }\end{array}$ \\
\hline S. epidermidis & 157 & 52.0 & 94.2 \\
S. hominis & 56 & 18.5 & 94.3 \\
S. haemolyticus & 52 & 17.2 & 94.1 \\
S. sciuri & 10 & 3.3 & 40.0 \\
S. warneri & 9 & 3.0 & 75.0 \\
S. saprophyticus & 6 & 2.0 & 90.0 \\
S. cohnii cohnii & 4 & 1.3 & 50.0 \\
S. simulans & 3 & 1.0 & 66.6 \\
S. capitis capitis & 2 & 0.6 & 50.0 \\
S. xylosus & 1 & 0.3 & 100 \\
S. capitis ureolyticus & 1 & 0.3 & 0 \\
S. cohnii urealyticum & 1 & 0.3 & 100 \\
\hline
\end{tabular}

TABLE II

Resistance of coagulase-negative Staphylococcus spp.

\begin{tabular}{lcc}
\hline & \multicolumn{2}{c}{ Resistance } \\
Test & n / Total & $\%$ \\
\hline Oxacillin, brand1 & $248 / 302$ & 82.1 \\
Oxacillin, brand 2 & $271 / 302$ & 89.7 \\
Cefoxitin, brand 1 & $154 / 302$ & 50.9 \\
Cefoxitin, brand 2 & $264 / 302$ & 87.4 \\
Oxacillin MIC & $273 / 302$ & 90.4 \\
mecA gene & $266 / 302$ & 88.1 \\
\hline
\end{tabular}

\section{DISCUSSION}

Considering that the treatment of staphylococcal infections depends on the methicillin resistance (Chandran \& Rennie 2005), and that this is habitually based upon the susceptibility to oxacillin and cefoxitin disks, the inconsistency of results may have important implications on inadequate therapy. It is important to point out that mortality rates in non-treated patients with bacteremia are greater than those of patients receiving adequate antimicrobial therapy (Weinstein et al. 1997) and that falsepositive resistance accounts for increased of costs, additional clinical work, request of more cultures, and unnecessary use of antibiotics such as vancomycin (Chandran \& Rennie 2005). 
TABLE III

Discordant results of susceptibility tests (disks brand 2)

\begin{tabular}{|c|c|c|c|c|}
\hline \multirow{2}{*}{$\begin{array}{l}\text { Staphylococcus } \\
\text { species }\end{array}$} & \multirow[b]{2}{*}{$m e c A$ gene } & \multirow{2}{*}{$\begin{array}{c}\text { Oxacillin } \\
\text { MIC }(\mu \mathrm{g} / \mathrm{ml})\end{array}$} & \multicolumn{2}{|c|}{ Disk diffusion } \\
\hline & & & Oxacillin & Cefoxitin \\
\hline S. sciuri & Negative & $\mathrm{R}(0.5)$ & $\mathrm{R}$ & $\mathrm{R}$ \\
\hline S. sciuri & Negative & $\mathrm{R}(0.5)$ & $\mathrm{R}$ & $\mathrm{S}$ \\
\hline S. sciuri & Negative & $\mathrm{R}(0.5)$ & $\mathrm{R}$ & $\mathrm{S}$ \\
\hline S. sciuri & Negative & $\mathrm{R}(0.5)$ & $\mathrm{R}$ & $\mathrm{S}$ \\
\hline S. cohnii cohnii & Negative & $\mathrm{R}(0.5)$ & $\mathrm{R}$ & $\mathrm{S}$ \\
\hline S. saprophyticus & Negative & $\mathrm{R}(0.5)$ & $\mathrm{R}$ & $\mathrm{S}$ \\
\hline S. cohnii cohnii & Negative & $\mathrm{R}(0.5)$ & $\mathrm{S}$ & $\mathrm{S}$ \\
\hline S. epidermidis & Negative & $\mathrm{R}(>4)$ & $\mathrm{S}$ & $\mathrm{S}$ \\
\hline S. sciuri & Negative & $\mathrm{S}(0.25)$ & $\mathrm{R}$ & $\mathrm{S}$ \\
\hline S. sciuri & Negative & $\mathrm{S}(0.25)$ & $\mathrm{R}$ & $\mathrm{S}$ \\
\hline S. haemolyticus & Positive & $\mathrm{R}(2)$ & $\mathrm{S}$ & $\mathrm{R}$ \\
\hline S. epidermidis & Positive & $\mathrm{R}(>4)$ & $\mathrm{S}$ & $\mathrm{R}$ \\
\hline S. cohnii cohnii & Positive & $\mathrm{R}(0.5)$ & $\mathrm{R}$ & $\mathrm{S}$ \\
\hline S. hominis hominis & Positive & $\mathrm{R}(>4)$ & $\mathrm{R}$ & $\mathrm{S}$ \\
\hline
\end{tabular}

R: resistant; S: susceptible; MIC: minimum inhibitory concentration.

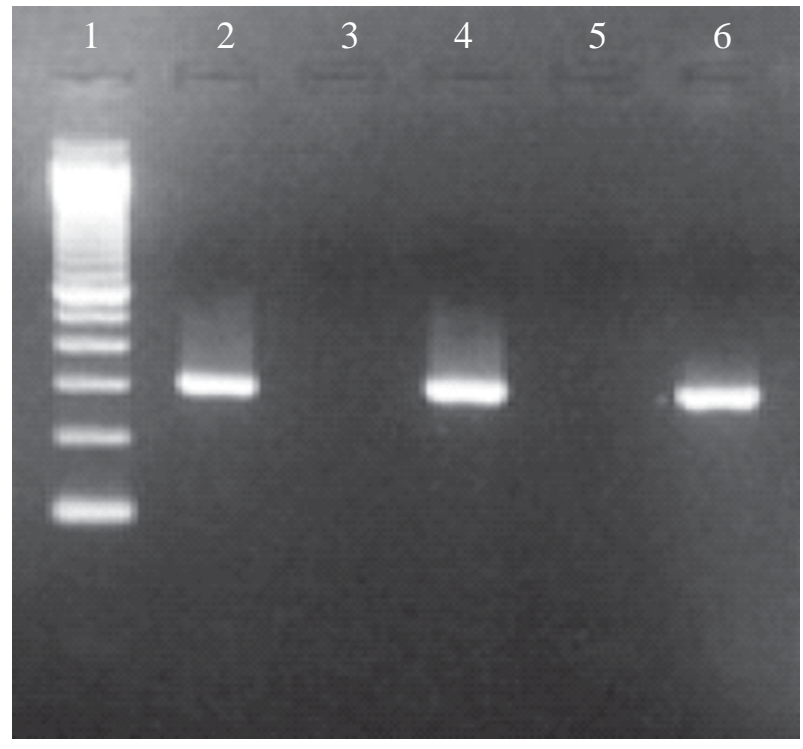

Polymerase chain reation detection of $m e c A$ gene of three isolates with discrepancy regarding oxacillin disk. Lanes - 1: molecular size markers (1100 bp); 2: positive control (310-bp band obtained with DNA from Staphylococcus aureus reference strain ATCC 33591); 3: negative control (S. aureus ATCC 25923); 4: clinical isolate (310-bp band obtained with DNA from $S$. haemolyticus); 5 : clinical isolate (absence of band with DNA from $S$. sciuri); 6: clinical isolate (310-bp band obtained with DNA from $S$. epidermidis).

The rates of resistance detected with oxacillin MIC determination were greater than those obtained with any other methods, but the majority of discrepant isolates displayed lower MIC $(0.5 \mu \mathrm{g} / \mathrm{ml})$. In fact borderline CoNS isolates are more difficult to detect, and changes in the interpretative zone diameters of cefoxitin have been suggested (Frigatto et al. 2005).
The brands used for the disk susceptibility tests are among those most common used in laboratories of routine microbiology as well as in some research (Sejas et al. 2003, Palazzo \& Darini 2006). Brand 1 is cheaper than brand 2 which displayed a better performance. It is important to point out that disks of cefoxitin brand 1 failed to detect resistance in many isolates, and that they are recommended by CLSI to detect resistance in CoNS.

Depending on the brand, cefoxitin disks showed distinct results: with brand 1 disks, only $60.9 \%$ of the resistant isolates detected by mecA gene were linked. On the other hand, brand 2 displayed higher concordant results $(98.7 \%)$, either among susceptible or resistant isolates. Oxacillin disks results were less divergent. The major difference of results between the two brands makes complex universal evaluations. We observed that brand 2 showed good correlation between MIC of oxacillin and disk diffusion (98.5\% for oxacillin and $97.8 \%$ for cefoxitin).

Cefoxitin disks of brand 2 displayed concordant results with mecA gene in 298/302 cases, failing to detect resistance in three isolates and susceptibility in one. Oxacillin disk results were concordant in 291 isolates, and also failed to detected resistance in three cases, but the failure to detect susceptibility was greater (eight cases). This data indicates the value of high-quality disks, as already pointed, and corroborates recommendations (Swenson \& Tenover 2005, CLSI 2006), to increase the sensitivity and specificity of the cefoxitin disk to detected oxacillin hetero-resistance.

High discrepancy of results between MIC of oxacillin and $m e c A$ gene was observed. Considering mecA gene as standard, eight isolates were false-positive, seven of them displayed a MIC of $0.5 \mu \mathrm{g} / \mathrm{ml}$, contrasting with a higher MIC of oxacillin ( $>4 \mu \mathrm{g} / \mathrm{ml}$ ) observed in the majority of concordant resistance cases. This may be due to the fact that the detection of resistance in iso- 
lates that displayed higher MIC is easier than among those with MIC closer to the susceptibility values. It must be pointed that we also observed discrepancy in the results of one isolate with MIC of $2 \mu \mathrm{g} / \mathrm{ml}$.

Given that mecA gene is present in the majority of the methicillin-resistant Staphylococcus (MRS), it can be considered the best method, even though discrepancies have been reported in CoNS isolates (Swenson \& Tenover 2005, Palazzo \& Darini 2006). Isolates with negative results for mecA gene and MIC for oxacillin $>0.5 \mu \mathrm{g} / \mathrm{ml}$ displayed controversial results in disk diffusion tests. For uncommon species such as $S$. cohnii and $S$. warneri, this is not surprisingly (Hussain et al. 2000, Palazzo \& Darini 2006). Resistance to oxacillin, without mecA gene, may be due to the overproduction or overexpression of penicillinase, or by alteration of other penicillin-binding proteins (Caierão et al. 2004).

The disk diffusion test proposed by Kirby-Bauer is one of the most used methods in Brazilian clinical laboratories for determination of bacterial susceptibility. It has already been pointed the importance of the quality of the disks (Sejas et al. 2003). Our study indicated that antimicrobial susceptibility test according to CLSI and the use of qualified disks (brand 2) led to reliable results, although in a few cases the disks were powerless to provide an adequate response, and corroborates the idea that the use of more methods or tests improves the detection of resistance (Frigatto et al. 2005).

Hussain et al. (2000) investigated the correlation of oxacillin MIC with presence of $m e c A$ by PCR and pointed that the breakpoints for oxacillin correctly categorize the most frequent CoNS but can not succeed for less common species. Our study also observed discrepancy among the less frequent CoNS. According to Skov et al. (2005), false resistance among CoNS is expected to be present in 1 to $9 \%$ of the cases. Although no technique alone display $100 \%$ of sensitivity and specificity to detect oxacillin resistance among CoNS, the combination of disk diffusion and oxacillin MIC can reduce failure to detection of such resistance.

Although cefoxitin is recommended by CLSI to detect oxacillin resistance in staphylococci, Frigatto et al. (2005) observed discrepant results between oxacillin and cefoxitin disks among S. epidermidis, and emphasized the importance of using molecular techniques to confirm disk diffusion results. Palazzo and Darini (2006) reported that the use of both cefoxitin disks and MIC of oxacillin may reduce errors in the detection of resistance to methicillin. While in our study we observed better concordance of mecA gene with cefoxitin disks rather than with oxacillin disks, we also observed some cases in which only oxacillin disk detect resistance. For this reason we consider important to perform tests with both disks for the moment.

Laboratories of microbiology need rapid, sensitive, and specific techniques to detect bacterial resistance. Molecular methods were recently introduced to improve results, but for most of the clinical laboratories these techniques are still too expensive. Disk susceptibility tests are widely available and the use of excellent quality disks can ensure results to properly guide antimicrobial therapy.

\section{ACKNOWLEDGMENTS}

To the technical team of the Laboratory of Gram-positive Cocci of the Fundação Faculdade Federal de Ciências Médicas de Porto Alegre.

\section{REFERENCES}

Bannerman TL 2003. Staphylococcus, Micrococcus, and other catalase-positive cocci that grow aerobically. In PR Murray, EJ Baron, JH Jorgensen, MA Pfaller, RH Yolken, Manual of Clinical Microbiology, American Society Microbiology, Washington, p. 384-404.

Beekmann SE, Diekema DJ, Chapin KC 2003. Effects of rapid detection of bloodstream infections on length of hospitalization and hospital charges. J Clin Microbiol 41: 3119-3125.

Caierão J, Musskopf M, Superti S, Roesch E, Dias CG, d'Azevedo PA 2004. Evaluation of phenotypic methods for methicillin resistance characterization in coagulase-negative Staphylococci (CNS). J Med Microbiol 53: 1195-1199.

Cauwelier B, Gordts B, Descheemaecker, Van Landuyt H 2004. Evaluation of a disk diffusion method with cefoxitin $(30 \mu \mathrm{g})$ for detection of methicillin-resistant Staphylococcus aureus. Eur J Clin Microbiol 6: 389-392.

Chambers HF 1997. Methicillin resistance in Staphylococci: molecular and biochemical basis and clinical implications. Clin Microbiol Rev 10: 781-791.

Chandran AU, Rennie R 2005. Routine antimicrobial susceptibility testing of coagulase-negative Staphylococci isolated from blood cultures: is it necessary? Clin Microbiol Infect Dis 11: 1037-1040.

CLSI/NCCLS-Clinical and Laboratory Standards Institute 2006. Performance Standards for Antimicrobial Susceptibility Testing - Sixteenth Informational Supplement M1000- S16. S, Wayne, PA, US.

Felten A, Grandry B, Lagrange PH, Casin I 2002. Evaluation of three techniques for detection of low-level methicillin-resistant Staphylococcus aureus (MRSA): a Disk Diffusion Method with cefoxitin and moxalactam the Vitek 2 System, and the MRSA-Screen Latex Agglutination Test. J Clin Microbiol 40: 2766-2771.

Frigatto EAM, Machado AMO, Pignatari ACC, Gales AC 2005. Is the cefoxitin disk test reliable enough to detect oxacillin resistance in coagulase-negative Staphylococci? J Clin Microbiol 43: 2028-2029.

Hussain Z, Stoakes L, Massey V, Diagre D, Fitzgerald V, Sayed S, Lannigan R 2000. Correlation of oxacillin MIC with mecA carriage in coagulase-negative Staphylococci. J Clin Microbiol 38: 752-754.

Lindsay JA, Aravena-Román MA, Riley TV 1993. Identification of Staphylococcus epidermidis and Staphylococcus hominis from blood cultures by testing susceptibility to desferrioxamine. Eur J Clin Microbiol Infect Dis 12: 127-131.

Mendes ER, Reis AO, Gales AC, Jones RN, Sader HS 2003. Ability of Latin America laboratories to detect antimicrobial resistance patterns: experience of the SENTRY Antimicrobial Surveillance Program (1997-2000). Braz J Infect Dis 7: 282-289. 
Nunes APF, Teixeira LM, Iorio NLP, Bastos CCR, Fonseca LS, Souto-Padrón T, Santos KRN 2006. Heterogeneous resistance to vancomycin in Staphyloccus epidermidis, Staphylococcus haemolyticus and Staphylococcus warneri clinical strains: characterization of glycopeptide susceptibility profiles and cell wall thickening. Int J Antimicrob Agents 27: 307-315.

Nunes ELC, Santos KRN, Mondino PJJ, Bastos MCF, Giambiagide Marval M 1999. Detection of ileS-2 gene enconding mupirocin resistance in methicilin-resistant Staphylococcus aureus by multiplex PCR. Diagn Microbiol Infect Dis 34: $77-81$.

Oliveira GA, Dell' Aquila AM, Masiero RL, Levy CE, Gomes MS, Cui L, Hiramatsu K, Mamizuka EM 2001. Isolation in Brazil of nosocomial Staphylococcus aureus with reduced susceptibility to vancomycin. Infect Control Hosp Epidemiol 22: 443-448.

Palazzo ICV, Darini ALC 2006. Evaluation of methods for detecting oxacillin resistance in coagulase-negative Staphylococci including cefoxitin disc diffusion. FEMS Microbiol Lett 257: 299-305.

Pottumarthy S, Fritsche TR, Jones RN 2005. Evaluation of alternative disk diffusion methods for detecting $m e c A$-mediated oxacillin resistance in an international collection of Staphylococci: validation report from the SENTRY Antimicrobial Surveillance Program. Diag Microbiol Infect Dis 51: 57-62.

Rosco - Diatabs ${ }^{\circledR}$ Diagnostic Tablets for identification 2000. Guideline A/S Rosco: 17-31.
Sader HS, Gales AC, Pfaller MA, Mendes RE, Zocolli C, Barth AL, Jones RN 2001. Pathogen frequency and resistance patterns in Brazilian hospitals: summary of results from three years of the SENTRY Antimicrobial Surveillance Program. Braz J Infect Dis 5: 200-214.

Sejas LM, Silbert S, Reis AO, Sader HS 2003. Avaliação da qualidade dos discos com antimicrobianos para testes de disco-difusão disponíveis comercialmente no Brasil. J Bras Patol e Med Labor 39: 27-35.

Skov R, Smyth R, Larsen AR, Frimodt-Møller N, Kahlmeter G 2005. Evaluation of cefoxitin 5 and $10 \mu \mathrm{g}$ discs for the detection of methicillin resistance in Staphylococci. J Antimicrob Chemother 55: 157-161.

Swenson JM, Tenover FC 2005. Results of disk diffusion testing with cefoxitin correlate with presence of mecA in Staphylococcus spp. J Clin Microbiol 43: 3818-3823.

Vannuffel P, Gigi J, Ezzedine H, Vandercam B, Delmme M, Wanters G, Gala JL 1998. Specific detection of methicilin resistant Staphylococcus species by multiplex PCR. J Clin Microbiol 33: 2864-2867.

Weinstein MP, Towns ML, Quartey SM, Mirret S, Reimer LG, Parmigiani G, Reller LB 1997. The clinical significance of positive blood cultures in the 1990s: a prospective comprehensive evaluation of the microbiology, epidemiology, and outcome of bacteremia and fungemia in adults. Clin Infect Dis 24: 584-602. 
